\title{
INVESTIGATION ON CIRCULAR MAPPING BY FMCW-SAR ON SMALL AIRPLANES
}

\author{
S. Palm ${ }^{\mathrm{a}, \mathrm{b}}$, A. Maresch ${ }^{\mathrm{a}}, \mathrm{U} \cdot$ Stilla ${ }^{\mathrm{b}}$ \\ ${ }^{a}$ Fraunhofer Institute for High Frequency Physics and Radar Techniques FHR, 53343 Wachtberg, Germany - \\ stephan.palm@fhr.fraunhofer.de \\ ${ }^{\mathrm{b}}$ Photogrammetry \& Remote Sensing, Technische Universitaet Muenchen (TUM), Munich, Germany \\ stilla@tum.de
}

KEY WORDS: Circular FMCW SAR, Nonlinear Flight Tracks, Beam Stabilization, UAV, mmWave, Antenna Steering

\begin{abstract}
:
The evaluation of local damages after natural disasters by using remote sensing demands for flexible platforms as well as sensor systems, which guarantee both weather- and daylight-independence. Due to the fact of small energy consumption, small size and light weight millimeter-wave FMCW radar sensors on small airplanes are very promising for this task. Especially in urban environments the side looking SAR geometry causes shadowing and foreshortening effects, which lead to gaps in the reconstructed scene and misinterpretation. Multiple views from different aspect angles can reduce the shadowing effects but especially in unknown areas the best line of sight cannot be investigated in advance. This is achievable by perfoming circular flight trajectories. However, as millimeter-wave FMCW radar sensors typically have very narrow beams and small airplanes are sensitive to air turbulences, a beam stabilization method is necessary to provide full illumination of the target scene. In this paper the calculations for system specific optimal flight parameters are demonstrated and validated by simulations. The impact of air turbulences, causing angular deviations to the sensor and thus misplacement of the main beam lobe on the ground are explored by experimental data performed with our SUMATRA system. The effects of a potential mechanical beam stabilization are visualized and requirements for such a system are formulated. Our experiments show that for typical flight conditions a stabilized platform is well suitable to stabilize a narrow radar beam in order to keep a target scene constantly illuminated over a full circular trajectory. Typically these stabilized platforms can handle angular corrections in all three geometries (pitch, roll, squint) of up to $12^{\circ}-15^{\circ}$ by a speed of $15^{\circ}$ per second. Therefore a more cost intensive full gimbal system which is known to be used in optical applications and which can handle a full $360^{\circ}$ tracking is not neccessarily needed.
\end{abstract}

\section{INTRODUCTION}

The evaluation of local damages after natural disasters by using remote sensing demands for flexible platforms as well as sensor systems, which guarantee both weather- and daylight-independence. Radar sensors on small manned or unmanned airplanes are well suited for those characteristics. Due to the fact of small energy consumption, small size and light weight millimeter-wave FMCW radar sensors are very promising for this task. Typically, image generating radar systems exploit the SAR principle using a side looking geometry. Especially in urban areas this geometry causes shadowing and layover effects, which leads to gaps in the mapped scene and problems in reconstruction (Stilla et al., 2003). Several authors have shown that multiple views from different aspect angles (azimut) of airborne systems can reduce the shadowed areas for reconstruction of roads (Stilla and Hedman, 2010), digital surface models (DSM) (Schmitt and Stilla, 2011) and buildings (Thiele et al., 2010). Different viewing angles in elevation allows to overcome the layover problem (Schmitt and Stilla, 2013),(Schmitt and Stilla, (in press)). For known areas the optimal trajectories of a flight campaign for minimizing the disturbing effects can be calculated in advance (Soergel et al., 2003), (Stilla et al., 2004). But, in case of unknown terrain the best lines of sight cannot be investigated in advance. Compared with the classical methodology of processing approximately linear flight tracks it seems reasonable to consider as many line of sights as possible to fill the gaps.

This is achievable by performing circular flight trajectories (hyperaspect or full-aspect) over the interesting scene. Due to the fact of the nonlinear flight path this trajectory however demands specific conditions for the recording as well as the beam stabilization and requires the development of new processing and analysis algorithms. The first author introducing the SAR processing approach of circular trajectories was (Soumekh, 1996), experiments with steerable antennas were performed in L, P and X-band (Cantalloube and Colin, 2006), (Cantalloube et al., 2007). Others considered the more general case of nonlinear flight tracks (Frey et al., 2009). DEM reconstruction over a circular dataset was demonstrated by (Palm et al., 2012).

The objective of this paper and the conducted experiments go in two directions. As typically the range of small millimeterwave FMCW radar sensors is limited compared to pulsed systems (Charvat and Kempel, 2006), the possibility of flying such small circular trajectories has to be explored. More important is the investigation on the physical requierements which are essential to stabilize a narrow beam on a defined target given the circular SAR geometry by using small airplanes. Such planes are usually high sensitive to air turbulences and thus a beam stabilization is expected to be necessary. In connection with airborne sensor stabilization, especially carriers of optical sensors, such as photo or video cameras, are known to use gimbal systems which allow for a full $360^{\circ}$ coverage. These systems are comparatively expensive and often very small. As alternative, a stabilized platform, which is less expensive but only shows partial angular corrections, usually in between $\pm 15^{\circ}$, can be used. As the decision for a system depends on the experimental results, we therefore started investigations to specify requirements on the physical parameters of the circular flight trajectories, the beam stabilization platform and the expected SAR raw data acquisition and geometry to define next processing steps.

\section{TRAJECTORY CALCULATION}

To calculate an ideal circular flight path with constant forward speed and height, the given physical parameters and limits of the radar sensor and of the small airplane have to be taken into account. Typical SAR-systems depend on a side looking geometry 
in which the radar is operated perpendicular to the flight direction under a certain depression angle $\phi$, which is indicated in figure 1a. For all following vectors $\vec{\omega}$ we apply: $\omega=|\vec{\omega}|$. To keep the airplane at a constant speed and height in this geometry only the gravitational force $\vec{F}_{G}=m \cdot \vec{g}$, where $\mathrm{m}$ is the mass and $\vec{g}$ the acceleration of gravity, has to be compensated by the plane's forward velocity. In a circular geometry as indicated in figure $1 \mathrm{~b}$, the plane additionally has to compensate for the centripetal force $\vec{F}_{Z F}=m \cdot \overrightarrow{a_{z}}$, with $\overrightarrow{a_{z}}$ as centrifugal acceleration, which acts perpendicular to the instantaneous flight direction. Usually the constant flight velocity $v$ - which depends on the type of airplane and defines the pulse repetition rate (PRF) in azimuth - and the mean target to range distance $r_{m i d}$ can only vary in certain typespecific parameters and are therefore considered as fixed values. The same applies for the depression angle $\phi$ which is assumed to be fixed. Given these three variables $v, r_{\text {mid }}$ and $\phi$, the roll angle $\alpha$ involving the flight height $h_{1}>h_{2}$ as last independent variable can be calculated.

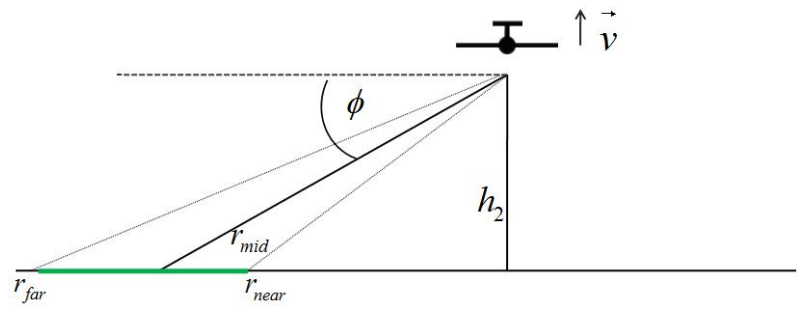

(a) Stripmap Geometry

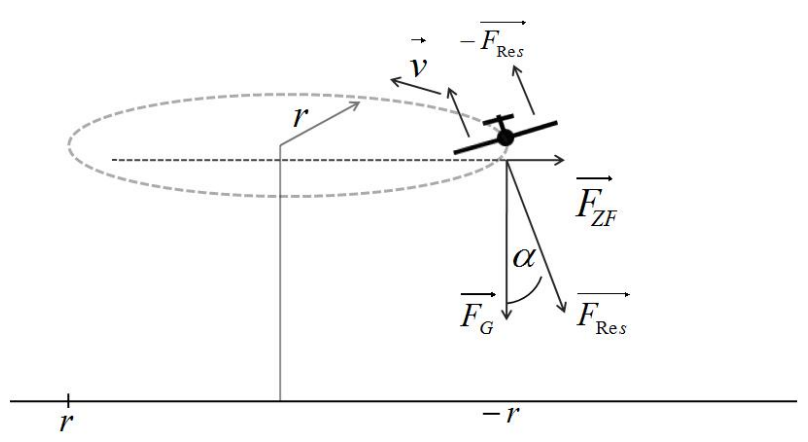

(b) Physical Forces

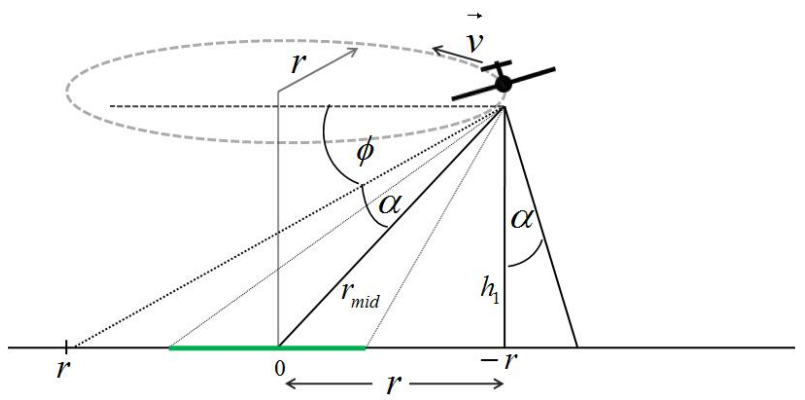

(c) Circular Geometry

Figure 1: SAR Geometry

From figure $1 \mathrm{~b}$ and $1 \mathrm{c}$ the roll angle $\alpha>0$ is simply expressed as the angle resulting from the two accelerations the plane has to compensate for. Therefore

$$
\tan (\alpha)=\frac{a_{z}}{g}
$$

where $a_{z}$ is the centrifugal acceleration and $g$ is the acceleration of gravity both affecting the plane. With $r>0$ as the circle's radius, $\cos (\alpha+\phi) \neq 0$ and

$$
a_{z}=\frac{v^{2}}{r}
$$

it follows

$$
\tan (\alpha)=\frac{a_{z}}{g}=\frac{v^{2}}{r \cdot g}=\frac{v^{2}}{g \cdot r_{m i d} \cdot \cos (\alpha+\phi)}
$$

or

$$
\tan (\alpha) \cdot \cos (\alpha+\phi)=\frac{v^{2}}{g \cdot r_{\text {mid }}}
$$

The term on the right side of equation 4 is now a system specific, dimensionless constant. Given the flight parameters $v, \phi$ and $r_{\text {mid }}$ from Table 1 below, it becomes the red constant marked in figure 2. A possible solution for $\alpha$ is found by using a roll angle of $16^{\circ}$.

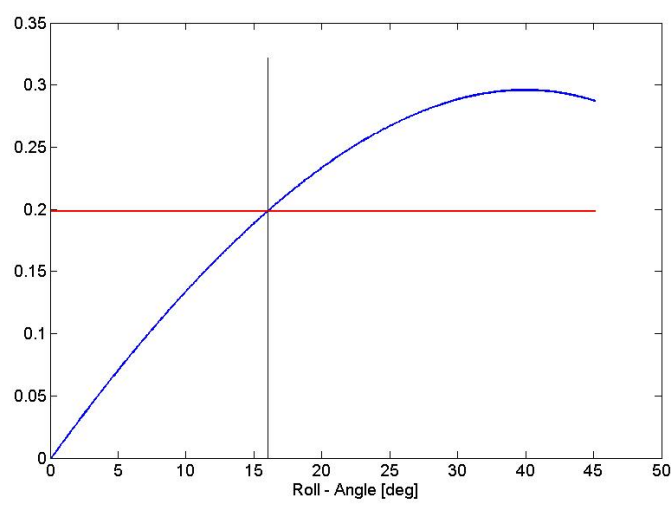

Figure 2: Roll angle $\alpha$ derivation

\section{BEAM FOOTPRINT CALCULATION}

The required beam stabilization parameters can be calculated using the inertial measuring unit's (IMU) data generated during the flight path. To project the potential beam on the earth geometry, knowledge of the three angular positions (pitch, roll and squint), the velocity components $V_{\text {North }}$ and $V_{\text {East }}$ and the flight height for each data package is neccessary. The heading direction $\varphi$ can then be calculated from the flight velocity $\vec{v}$ and the squint angle. In a three dimensional cartesian geometry as described in figure 3 , each sensor position can be expressed as a vector $\overrightarrow{r_{1}}$. To simplify the geometry, the origin of the coordinate system is set on the flat earth plain on which the radar beam is projected on. Thus the plain can consequently be parameterized by $\left(\vec{r}-\overrightarrow{r_{0}}\right) \cdot \vec{n}=0$, with $\overrightarrow{r_{0}}=(0,0,0)$ and $\vec{n}=(0,0,1)$. Further the projected beam can be calculated as an intersection of the ground plain with the look direction vector of the radar $\vec{a}$. As in typical SAR geometry the flight direction - or more accurate the heading direction $\varphi$ - is perpendicular to the sensor operation, the 


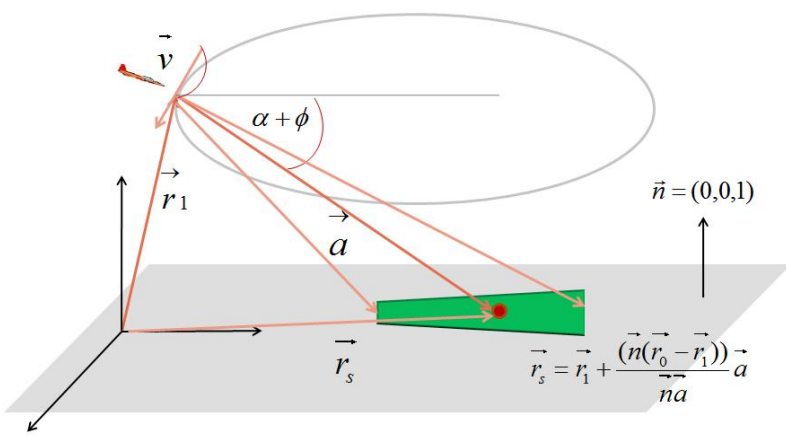

(a)

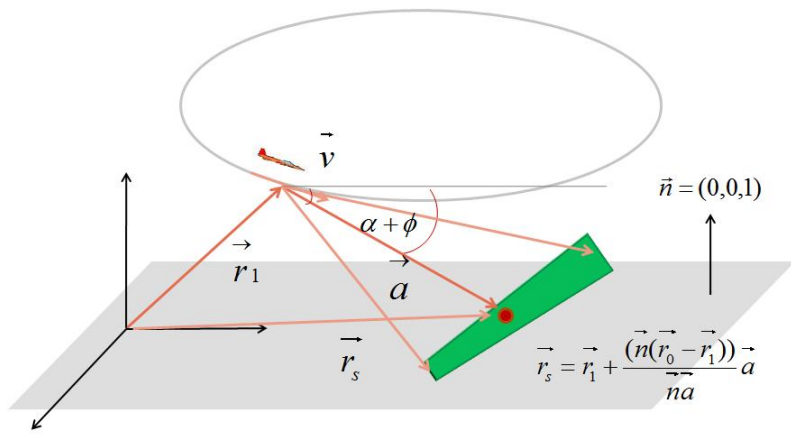

(b)

Figure 3: Circular SAR Geometry - Calculation of the instantaneous beam focus center

radar's look direction vector $\vec{a}$ for the beam focus point $\overrightarrow{r_{s}}$ can be calculated by

$$
\vec{a}=\left(\sin \left(\varphi-\frac{\pi}{2}\right), \cos \left(\varphi-\frac{\pi}{2}\right),-\tan (\alpha+\phi)\right)
$$

with $\alpha$ the roll angle and $\phi$ the depression angle, see figure 1 and 3. Consequently the intersection point and thus the focus center regarding the projected flat plain is defined by

$$
\overrightarrow{r_{s}}=\overrightarrow{r_{1}}+\frac{\vec{n} \cdot\left(\overrightarrow{r_{0}}-\overrightarrow{r_{1}}\right)}{\vec{n} \cdot \vec{a}} \cdot \vec{a}
$$

Similarly the intersection points $r_{\text {near }}$ and $r_{f a r}$ and thus the whole footprint can be calculated.

\subsection{Beam Stabilization}

The calculations in section 3 are neccessary to simulate and visualize the footprint on the projected plain and to calculate the deviation from the real beam center $\overrightarrow{r_{s}}$ compared to the optimal, ideal trajectory, in which the target center is constantly illuminated. As the real, approximately circular flight path will always show differences to the ideal path due to air turbulences, the sensor must mechanically be readjusted in its angular position to keep the target constantly illuminated. To define potential requirements for such a platform, the appropriate angular readjustments at each sensor position have been calculated by simulating a possible operation of such a system. Therefore, the individual impact of the three angular deviations (pitch, roll and squint) and their effect on the displacement of the radar footprint was separated and geometrical calculations are demonstrated below.

3.1.1 Pitch Stabilization: As the influence of the pitch angle regarding the beam misplacement and the angle variations are comparatively small, our calculations suppose that a beam stabilization mechanism is able to compensate for the resulting offset pitch angle. The three dimensional geometry can then be separated in a two dimensional geometry, regarding and separating the much more critical displacement in azimuth (squint angle) and in range (roll angle), which simplifies the model.

3.1.2 Squint Stabilization: To keep the focus point perfectly in the azimuth beam center, the target must be perpendicular to the flight - or more accurate heading direction $\varphi$. This is geometrically defined as the intersection angle $\psi$ of the two vectors, in our case $\overrightarrow{z_{1}}$, the difference between sensor position and the potential target, and $\overrightarrow{a_{1}}$, the heading direction, see figure $4 \mathrm{a}$. The $\mathrm{x}, \mathrm{y}$ coordinates of the beam focus point are $m_{x}$ and $m_{y}$, respectively. Given $\overrightarrow{z_{1}}=\left(m_{x}, m_{y}, h_{1}\right)-\overrightarrow{r_{1}}$ and $\overrightarrow{a_{1}}=(\sin (\varphi), \cos (\varphi), 0)$, with $\varphi$ the heading direction, we get:

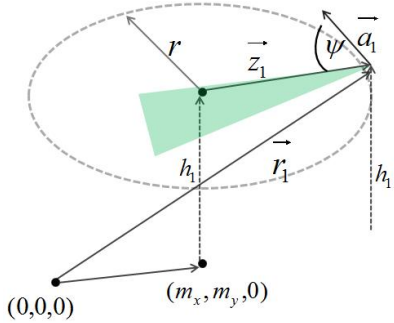

(a) Squint Angle

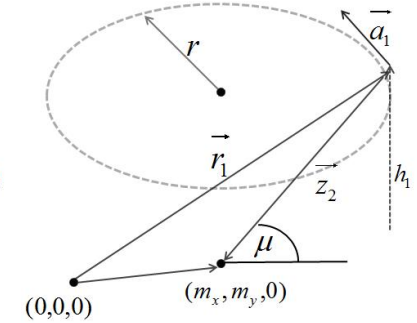

(b) Roll Angle
Figure 4: Calculation of optimal roll and squint angle

$$
\psi=\arccos \left(\frac{\overrightarrow{z_{1}} \cdot \overrightarrow{a_{1}}}{\left|\overrightarrow{z_{1}}\right| \cdot\left|\overrightarrow{a_{1}}\right|}\right)
$$

with $\psi$ being the angle between heading direction and target. With this information a beam stabilizing platform is able to readjust the angular position of the sensor to achieve the criterion $\psi=\frac{\pi}{2}$. Deviations from $\frac{\pi}{2}$ have to be corrected for and give information in which magnitude and degree of speed a sensor correction has to take place.

3.1.3 Roll Stabilization: The necessary beam correction in range can be achieved by readjusting the roll angle. Although modifying the sensor's roll angle does not change the distance between target and sensor, it influences the area the beam is illuminating on the ground. To calculate the ideal roll angle at each sensor position for the given circular flight path, the geometry slightly differs from the one used above to correct for the squint angle. The perfect angle to keep the target in the beam center is defined by the intersection angle $\mu$ between the vector $\overrightarrow{z_{2}}$ and the flat plain, see figure $4 \mathrm{~b}$. With $\overrightarrow{z_{2}}=\left(m_{x}, m_{y}, 0\right)-\overrightarrow{r_{1}}$ it follows

$$
\mu=\arcsin \left(\frac{\left|\vec{n} \cdot \overrightarrow{z_{2}}\right|}{|\vec{n}| \cdot\left|\overrightarrow{z_{2}}\right|}\right) .
$$

By defining the coordinates of a possible target center and knowing the sensor's position at each pulse, a constantly illuminated target center is consequently possible by stabilizing the three angular sensor directions according to the calculations shown.

\section{INSTRUMENTATION AND EXPERIMENT}

\subsection{Sensor Description}

The radar sensor frontend SUMATRA, developed and operated at Fraunhofer FHR, operates at $94 \mathrm{GHz}$ or $35 \mathrm{GHz}$, respectively, 
with a frequency bandwidth of up to $1 \mathrm{GHz}$ leading to a range resolution of $15 \mathrm{~cm}$ (Stanko et al., 2012). The SAR process requires either an ideal linear flight track or a very precise knowledge of the platforms movements. This typically means an accuracy of $\frac{\lambda}{10}$ for a complete aperture. Due to our small wavelength $\lambda$ of 3.2 $\mathrm{mm}$ at W-band, millimeter-wave SAR on small airplanes, which show high vulnerability to air turbulences, generally differs from conventional SAR as the corresponding accuracy of $0.32 \mathrm{~mm}$ over a full flight path is not feasible. Otherwise the small wavelength allows for coherent processing of very small apertures of only a few meters to achieve a range corresponding azimuth resolution. Consequently we use high focusing antennas with an azimuth beam width of only $1.5^{\circ}$ in W-band or $3.0^{\circ}$ at Ka-band, respectively, which further improves the signal to noise ratio due to the limited signal transmit power. At typical flight heights of $500-600 \mathrm{~m}$ this corresponds to an azimuth beam width of 17 $\mathrm{m}$ (W-band) or $35 \mathrm{~m}$ (Ka-band), respectively. This beam width is only partially used for SAR focussing. In normal stripmap mode we encounter already squint angles of up to $12^{\circ}$ due to air turbulences. When performing circular flight paths this variations are not acceptable as the beam footprint has to be stabilized with an accuracy of $0.75^{\circ}$ (W-band) or $1.50^{\circ}$ (Ka-band) in azimuth and $5^{\circ}$ in depression to keep the central target in the beam, even on ideal trajectories, which are not feasible. This very small beam width in azimuth results in only marginal tollerance for squint angle deviations, suggesting that beam stabilization in azimuth is most critical for our task.

\subsection{Experimental setup}

To specify the beam stabilization requirements we conducted test flights with our FMCW radar sensor SUMATRA and our IMU (AEROControl Specs, IGI, Kreuztal, n.d.) mounted on the ultralight aircraft DELPHIN. The pilot was given two different beam center coordinates and the calculated physical parameters to fly an approximately circular trajectory around the two targets. The sensor parameters and the flight specifications are shown in Table 1. We used a single corner on a field as first and a farm building as second ground target.

\begin{tabular}{|l|l|}
\hline Height above ground $h_{1}$ & $450 \mathrm{~m}$ \\
Radius $r$ & $437 \mathrm{~m}$ \\
Flight velocity $v$ & $35 \mathrm{~m} / \mathrm{s}$ \\
Depression angle $\phi$ & $30^{\circ}\left( \pm 5^{\circ}\right)$ \\
Roll angle $\alpha$ & $16^{\circ}$ \\
Mean target range $r_{\text {mid }}$ & $630 \mathrm{~m}$ \\
Carrier frequency $f_{0}$ & $35 \mathrm{GHz}$ \\
Azimuth beam width & $3^{\circ}$ \\
\hline
\end{tabular}

Table 1: Flight and Sensor Parameter

Analysis of the experimental flight data was done by processing the IMU data of the flights which is working with a data rate of $64 \mathrm{~Hz}$. Each IMU data sample was taken and the geometry of the radar beam - the footprint - on the projected ground plane was calculated and visualized as described in section 3. We then examined the individual impact of the three angular deviations (pitch, squint, roll) and the difference of the real trajectory compared to the ideal trajectory on the displacement of the radar footprint as described in Section 3.1.

As the deviations to the ideal circular trajectory cannot be corrected, we furthermore calculated the effects of operating with a possible beam stabilization platform on the real dataset. Given a certain time delay to take into account the mechanical steering, we simulated the effects of stabilizing each single angular displacement to keep the center of the footprint illuminating the target scene.

\section{FINDINGS}

\subsection{Beam Visualization}

The beam footprint visualization is demonstrated in figure 5. Each row shows three images taken from a video sequence while the plane is continuously moving forward on its trajectory. The real flight path is indicated in blue, the actual position and the allready covered distance of the plane is marked in red as well as the ideal focus point in the central image. The sensor's main lobe is visualized as green footprint and is continuously overlapped. To keep the video more clearly we only considered every tenth IMU data sample.

The first row (a-c) shows the calculated ideal circular flight path as described in section 2 and its projected radar beam. It uses the parameters of Table 1 and the $35 \mathrm{GHz}$ frontend. The target is constantly illuminated over the full circle.

The following rows show the experimental flight data and the impact to the footprint. The sensor's movement is approximately circular, indicating the pilot's ability to fly the defined trajectory. In the second row (d-f) the real untreated raw data is visualized. As expected, a roughly circular surface is mapped but the defined target area constantly moves out of the main beam lobe. A stable beam position is not achieved.

Row (g-i) indicates the effect of a beam stabilization in the roll angle as calculated in section 3.1.3. The partially strong roll angle deviations were compensated and the beam can be stabilized in range direction. The result is still inadequate.

The fourth line (j-1) shows the beam stabilization effects in the squint angle. The azimuth errors are compensated and the focus center is already nearly fully covered in the main beam lobe except when strong roll angle movements occur. However, the focus point does significantly move in range over time.

Finally the last row (m-o) indicates the effects of a possible beam stabilization platform in all three angular positions simultaneously. Although we use a time delay of 10 IMU data packages to compensate for the mechanical steering, the focus center is constantly illuminated by the main beam. Thus full aspect coverage is feasible.

\subsection{Stabilization Requirements}

The corresponding angular stabilization to achieve full aspect coverage is shown in figure 6 . The first row indicates roll angle, the second row squint and the third row pitch correction over the full trajectory. On the left side the total amount of stabilization in degree is plotted over the full trajectory, whereas on the right side the rate of angular change is visualized, namely delta-roll, deltasquint and delta-pitch. We used twice the time delay of 10 IMU data samples, corresponding to $0.32 \mathrm{sec}$, as reference. As expected, the amplitudes for the squint and roll angle corrections are the most critical ones. They lie roughly in between $-5^{\circ}$ to $+15^{\circ}$ for roll and $\pm 10^{\circ}$ for squint, however deviations in squint are much more crucial. The compensation rate for squint and pitch lies in between $\pm 1^{\circ}$, for roll between $\pm 2^{\circ}$ every 0.32 seconds.

\section{CONCLUSION}

The results demonstrate that although millimeter-wave FMCW radar sensors usually have a limited range compared to conventional pulsed systems, the possibility of using small airplanes allows for flying approximately circular trajectories with small radiuses at relatively low heights. However, due to the fact that 
International Archives of the Photogrammetry, Remote Sensing and Spatial Information Sciences,

Volume XL-1/W1, ISPRS Hannover Workshop 2013, 21 - 24 May 2013, Hannover, Germany

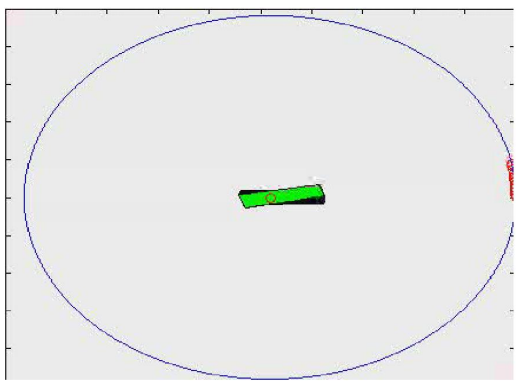

(a)

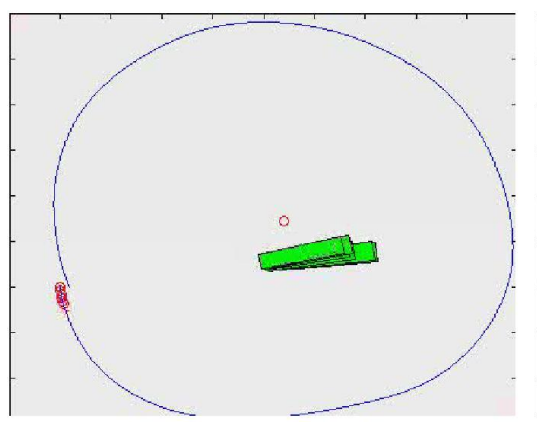

(d)

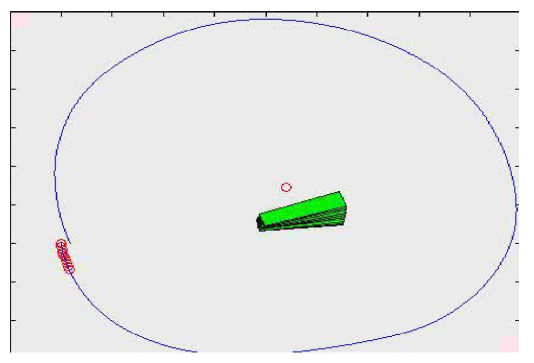

(g)

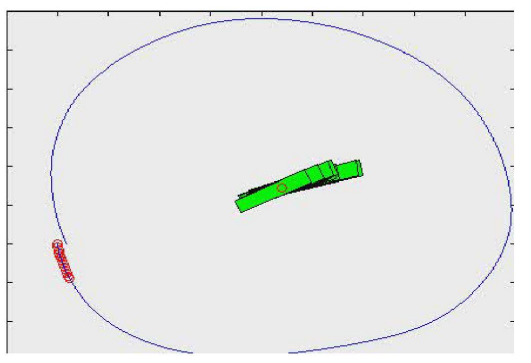

(j)

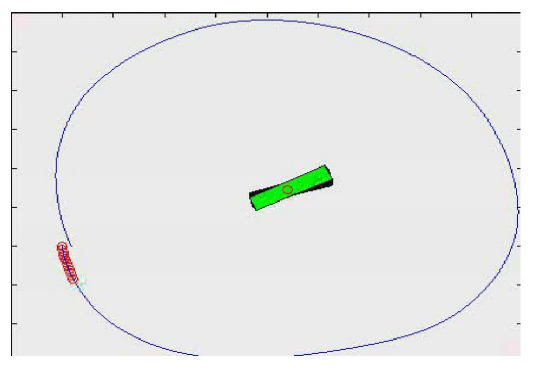

(m)

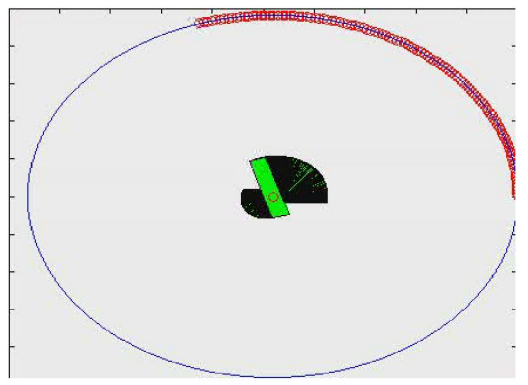

(b)

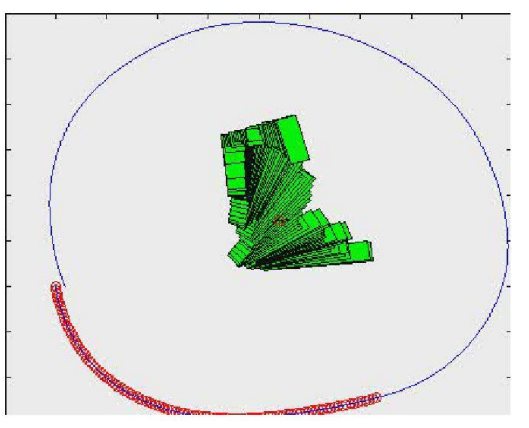

(e)

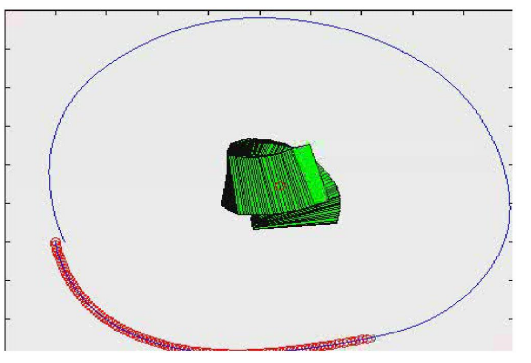

(h)

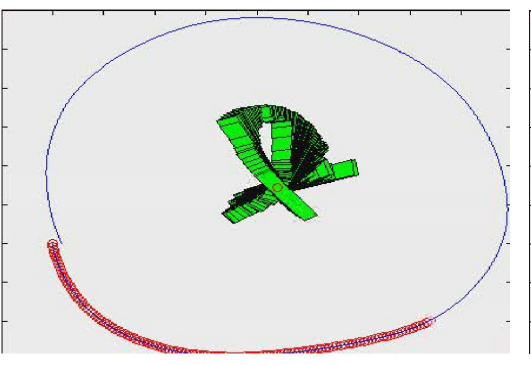

(k)

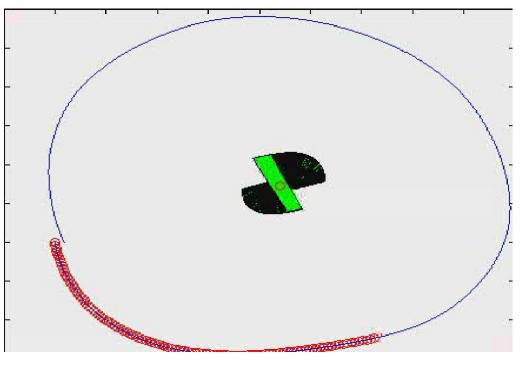

(n)

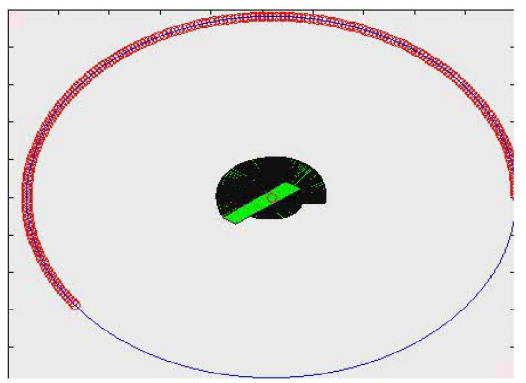

(c)

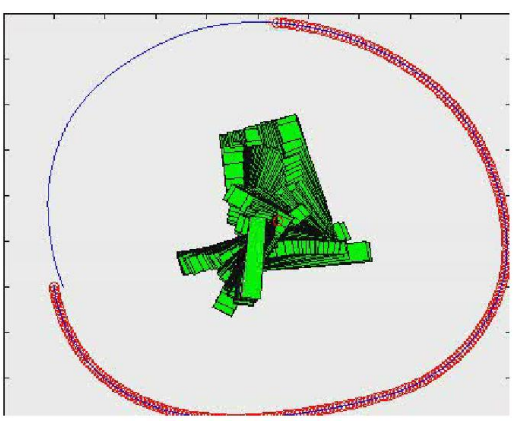

(f)

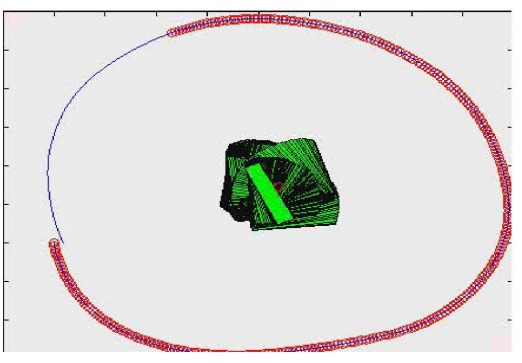

(i)

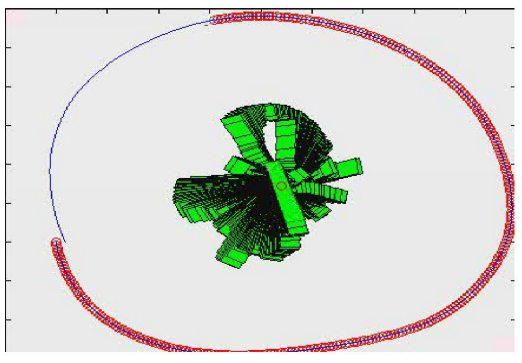

(1)

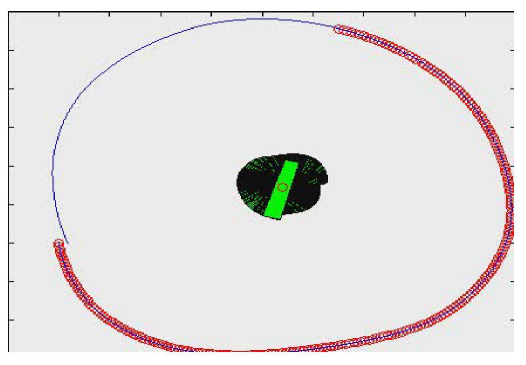

(o)

Figure 5: Flight and beam pattern visualization of experimental data. Images taken from a continuous video sequence from left to right. Flight trajectory is marked blue, actual position and already covered distance of airplane is marked red, instantaneous radar footprint is simulated green and continuously overlapped. (a-c):Ideal simulated circular trajectory, (d-f): Real experimental data, (g-i): Experimental data stabilized in roll angle, $(\mathrm{j}-1)$ : Experimental data stabilized in squint angle, $(\mathrm{m}-\mathrm{o})$ : Experimental data stabilized in pitch, squint and roll. 


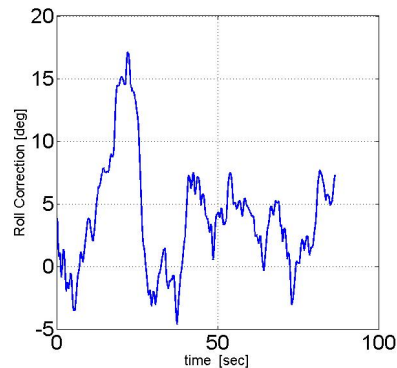

(a)

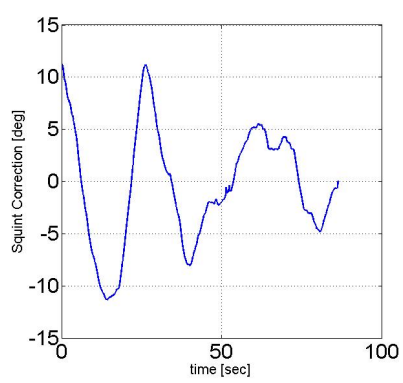

(c)

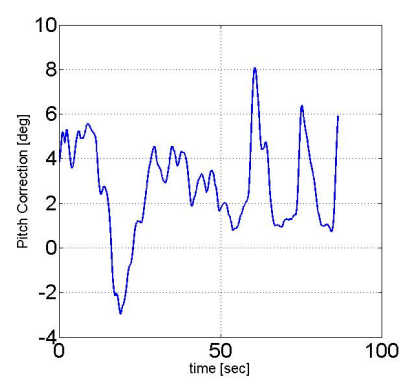

(e)

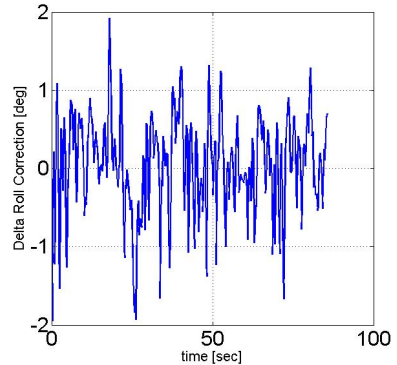

(b)

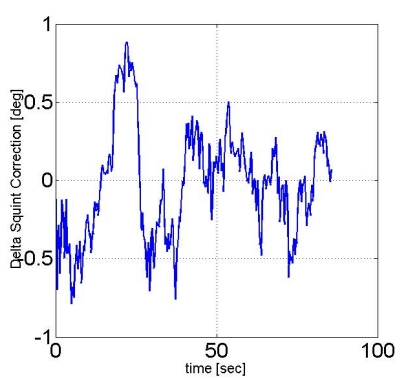

(d)

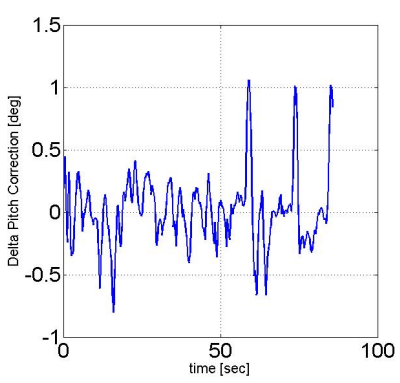

(f)
Figure 6: Stabilization Parameters for roll (a,b), squint (c,d) and pitch $(e, f)$ showing experimental data. Total rate of correction over full trajectory on the left side - total compensation rate on the right side.

typically high focusing antennas are used resulting in small azimuth beam width, the deviations caused by air turbulences and flight inaccuracies cause serious problems. A constantly illuminated target scene and thus a full aspect $360^{\circ}$ view is not feasible as the radar footprint is too sensitive for angular variances. As expected, the impact of the squint angle is most evident as the focus point continuously moves out of the main beam lobe.

Nevertheless, the results concerning the possible beam stabilization parameters are very promising. We could demonstrate that a stabilized platform, which is able to constantly readjust mechanically the angular position of the radar sensor is well suited to fulfill the full aspect task. An angular stabilization rate of up to $\pm 15^{\circ}$ and a compensation rate of $15^{\circ}$ per second, which usually specifies such systems, are well above the necessary values we calculated in our experiments. Thus, a more cost expensive full gimbal system seems not to be necessary.

Therefore, the next steps are the integration of the used radar frontend into a stabilzed platform, to further evaluate suitable circular SAR focusing techniques and to find strategies to merge the full aspect SAR images.

\section{REFERENCES}

AEROControl Specs, IGI, Kreuztal, n.d. http: //www.igi.eu/aerocontrol.html?file=tl_files/ IGI/Brochures/AEROcontrol/AEROcontrol_specs.pdf.

Cantalloube, H. and Colin, E., 2006. Airborne SAR imaging along a circular trajectory. In: EUSAR 6th. European Conference on Synthetic Aperture Radar, Dresden.

Cantalloube, H. M. J., Colin-Koeniguer, E. and Oriot, H., 2007. High resolution SAR imaging along circular trajectories. In: Geoscience and Remote Sensing Symposium, 2007. IGARSS 2007. IEEE International, pp. 850-853.

Charvat, G. and Kempel, L. C., 2006. Synthetic aperture radar imaging using a unique approach to frequency-modulated continuous-wave radar design. Antennas and Propagation Magazine, IEEE 48(1), pp. 171-177.

Frey, O., Magnard, C., Ruegg, M. and Meier, E., 2009. Focusing of airborne synthetic aperture radar data from highly nonlinear flight tracks. Geoscience and Remote Sensing, IEEE Transactions on 47(6), pp. 1844-1858.

Palm, S., Oriot, H. and Cantalloube, H., 2012. Radargrammetric DEM extraction over urban area using circular SAR imagery. Geoscience and Remote Sensing, IEEE Transactions on 50(11), pp. 4720-4725.

Schmitt, M. and Stilla, U., 2011. Fusion of airborne multi-aspect insar data by simultaneous backward geocoding. In: Proc. Joint Urban Remote Sensing Event (JURSE), pp. 53-56.

Schmitt, M. and Stilla, U., 2013. Compressive sensing based layover separation in airborne single pass multi-baseline InSAR data. IEEE Geoscience and Remote Sensing Letters 10 (2), pp. 313-317.

Schmitt, M. and Stilla, U., (in press). Adaptive multilooking of airborne single-pass multi-baseline InSAR stacks. IEEE Transactions on Geoscience and Remote Sensing.

Soergel, U., Schulz, K., Thoennessen, U. and Stilla, U., 2003. Event-driven SAR Data Acquisition in Urban Areas Using GIS. GeoBIT/GIS Journal for spatial information and decision making 12, pp. 32-37.

Soumekh, M., 1996. Reconnaissance with slant plane circular SAR imaging. Image Processing, IEEE Transactions on 5(8), pp. 1252-1265.

Stanko, S., Johannes, W., Sommer, R., Wahlen, A., Schroder, M. and Caris, M., 2012. SUMATRA - A UAV based miniaturized SAR System. EUSAR 9th. European Conference on Synthetic Aperture Radar 2012 pp. 437-440.

Stilla, U. and Hedman, K., 2010. Feature Fusion Based on Bayesian Network Theory for Automatic Road Extraction. Dordrecht: Springer, pp. 69-86.

Stilla, U., Michaelsen, E., Soergel, U., Hinz, S. and Ender, H. J., 2004. Airborne monitoring of vehicle activity in urban areas. In: International Archives of Photogrammetry and Remote Sensing, pp. 973-979.

Stilla, U., Soergel, U. and Thoennessen, U., 2003. Potential and limits of InSAR data for building reconstruction in built upareas. ISPRS Journal of photogrammetry and remote sensing 58, pp. 113-123.

Thiele, A., Cadario, E., Schulz, K. and Soergel, U., 2010. Analysis of Gable-Roofed Building Signature in Multiaspect InSAR Data. Geoscience and Remote Sensing Letters, IEEE 7(1), pp. 83-87. 